\title{
Acute limb ischemia following perineal reconstruction in lithotomy position: take-home message for plastic surgeons
}

\author{
Gianluca Sapino ${ }^{1}$, Sebastien Deglise ${ }^{2}$, Wassim Raffoul ${ }^{1}$, Pietro G. di Summa ${ }^{1}$ \\ Departments of ${ }^{1}$ Plastic, Reconstructive and Hand Surgery and ${ }^{2}$ Vascular Surgery, University Hospital of Lausanne (CHUV), University of \\ Lausanne, Lausanne, Switzerland
}

\begin{abstract}
Despite the extensive use of lithotomy position in several plastic surgery procedures, most reports regarding the related incidence of complications are presented in the urologic, gynecologic, and anesthesiologic fields. We present the case of a 54-year-old male patient. polytrauma patient who underwent internal iliac artery embolization leading to extensive gluteal necrosis requiring: debridement, abdominoperineal resection and composite anterolateral thigh flap reconstruction with prolonged lithotomy position. The patient presented lower limb ischemia briefly after surgical theater. A computed tomography scan revealed the obstruction of the left superficial femoral artery requiring emergency revascularization. Arterial thrombosis is a potentially devastating complication and plastic surgeons should be aware of the possible dangers when performing surgeries in prolonged lithotomy position. Preoperative detection of patients at high risks for developing complications should be performed in order to implement preventive measures and avoid potentially life-threatening sequelae.
\end{abstract}

Keywords Reconstructive surgical procedure / Surgery, plastic / Limb ischemia

\author{
Correspondence: Gianluca Sapino \\ Department of Plastic, Reconstructive \\ and Hand Surgery, Centre Hospitalier \\ Universitaire Vaudois (CHUV), Rue du \\ Bugnon 46, 1011 Lausanne, \\ Switzerland \\ Tel: +41-21-314-25-10 \\ Fax: +41-21-314-25-30 \\ E-mail: Gianluca.sapino@gmail.com
}

\section{INTRODUCTION}

The lithotomy position is commonly used to perform a wide range of operations, particularly in gynecologic, urologic and colorectal surgery [1]. For plastic surgeons, its role is mainly reserved to transgender surgery, perineal reconstruction and surgery of genitalia.

If some potentially catastrophic complications, such as deep venous thrombosis (DVT) or even compartmental syndrome of the limb, have been described and are well documented in literature $[2,3]$, acute arterial thrombosis in such setting is highly rare and only occasionally reported $[4,5]$. Indeed, possible implications of prolonged lithotomy position are probably underestimated by plastic surgeons in their common practice.

\section{CASE}

We report the case of a 54-year-old male patient with a recent history of polytrauma (1 month before), and was referred to our institute because of an extended perineal and left gluteal defect of $18 \times 25 \mathrm{~cm}$. Following the high energy trauma, the patient presented hemodynamic instability on a pelvis open book frac- 
ture requiring emergency external fixator of the pelvis and radiological embolization of the left iliac artery for life-threatening bleeding. This led to multiple abscess in the perineal/gluteal region due to necrotic tissues, which finally required an abdominoperineal amputation, and an important loss of perineal and left gluteal soft tissue coverage (Fig. 1). Furthermore, he developed a
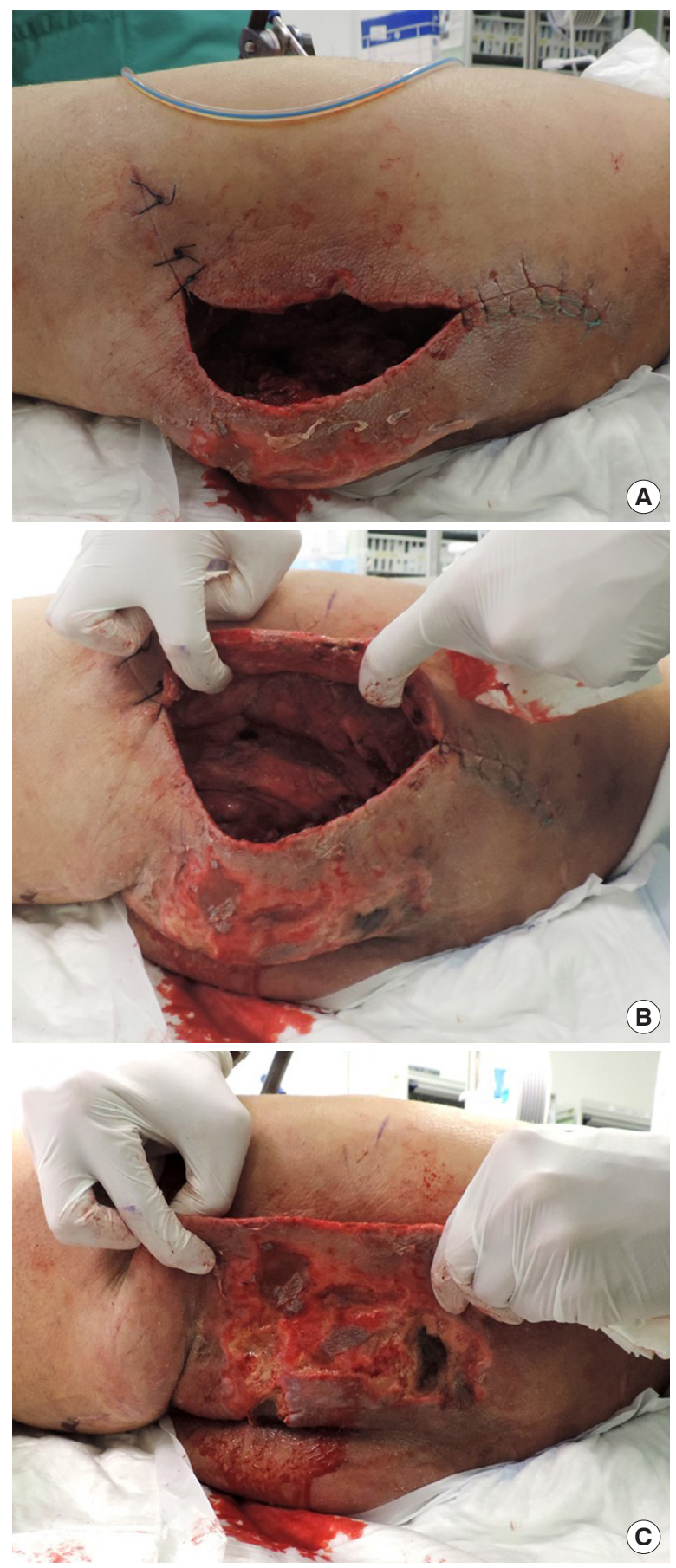

Fig. 1. A case of extended perineal and gluteal defect. The defect at the left gluteal/perineal region (A), with important dead space (B) and skin necrosis (C). bilateral DVT at the lower limbs with pulmonary embolism requiring inferior cava filter and therapeutic anticoagulation.

The reconstructive plan included a pedicled anterolateral thigh (ALT) flap extended to vastus lateralis (VL) muscle to obtain more bulk to fill the defect and cover the exposed left ischium. At surgery, patient had a body mass index of $23 \mathrm{~kg} / \mathrm{m}^{2}$. Preoperative blood test showed hemoglobin levels at $8.3 \mathrm{~g} / \mathrm{dL}$ and thrombocytes at $426 \times 10^{9} / \mathrm{L}$. Therapeutic anticoagulation (intravenous heparin) was stopped 6 hours prior to surgery with normal prothrombin time (100\%), and international normalized ratio [1]. With the patient in supine position the composite ALT-VL flap was harvested as previously described [6]. After flap raise, to provide fully access to the perineal and gluteal region, the patient was then positioned in high lithotomy position (Fig. 2). The pedicled ALT flap was transposed under the sartorius and the rectus femoris muscle to cover the perineal and gluteal area, passing posteriorly to the superficial femoral artery. The procedure lasted in total 5 hours with at least 2 hours in lithotomy position. Blood loss estimated was less than $500 \mathrm{~mL}$

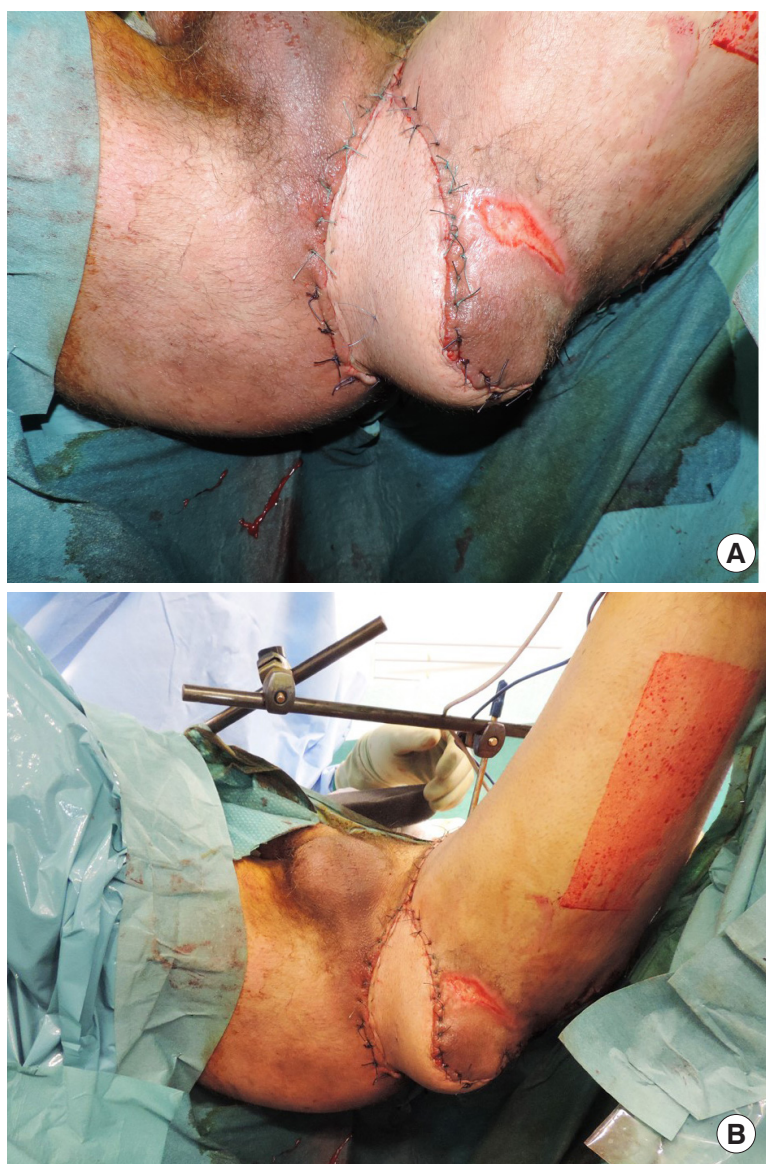

Fig. 2. Perineal reconstruction using an anterolateral thigh flap. After the harvesting, the pedicled composite anterolateral thigh flap+vastus lateralis muscle was used to cover the defect and fill the dead space (A) with the patient placed in high lithotomy position (B). 

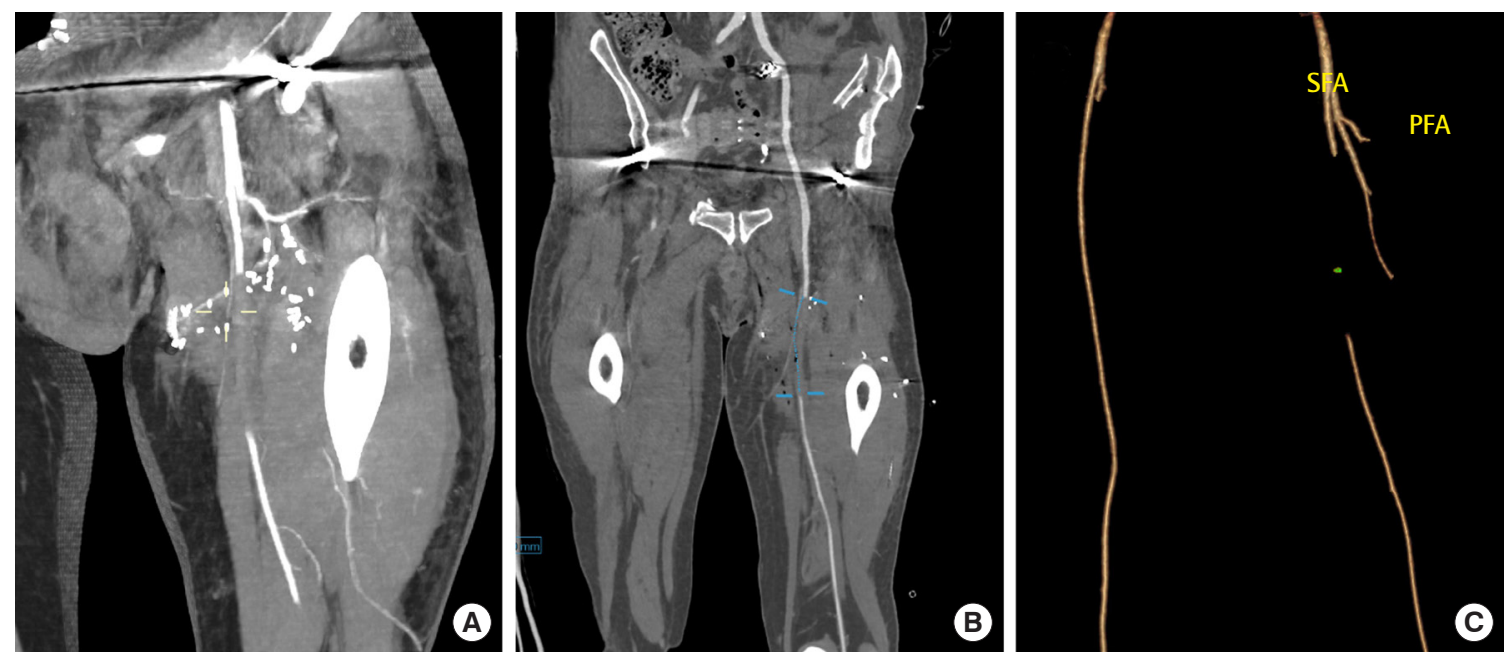

Fig. 3. Acute limb ischemia following lithotomy position. Angio-computed tomography (CT) performed in the immediate postoperative period, showing the transposed pedicle of the flap (descending branch of the lateral circumflex femoral artery) crossing the SFA towards medial direction (A). Arterial thrombus (over $8 \mathrm{~cm}$ ) of the SFA in the coronal angio-CT section (B) and 3-dimensional reconstruction (C). SFA, superficial femoral artery; PFA, profunda femoral artery.

and blood pressure was maintained stable during the whole surgery.

Two hours after surgery a pale and cold lower limb was noticed with no Doppler pulse at the foot, while flap showed normal appearance and appropriate capillary refill, speaking for an arterial occlusion distally to the depart of the lateral circumflex femoral artery. An emergency angio-computed tomography scan was performed, showing an $8 \mathrm{~cm}$ thrombus at left femoral artery (Fig. 3). An emergency Fogarty procedure was necessary to remove the clot and re-establish optimal flow to the limb. Postoperative outcomes were otherwise uneventful with effective flap coverage and no limb sequelae.

\section{DISCUSSION}

Despite his rarity in the perioperative period, acute artery thrombosis in lithotomy position represents a potentially lifethreatening complication. After attentive literature search, most reports regarding the incidence of complications related to the lithotomy position were presented in the urologic, gynecologic and anesthesiologic fields [7-9]. Despite the potentially devastating consequences, no report was found in plastic surgery journals.

Being rare, the incidence of femoro-iliac artery thrombosis during pelvic surgery is unknown: as reported by Sahara et al. [4] only nine case reports have been published so far. According to Kikuchi and Maeda [10] prolonged surgery of 5 hours or more, lithotomy position, low blood pressure, low body temperature, dehydration, compression of the lower extremities by the surgeon, are among the most frequently involved perioperative- related factors in the development of a compartment syndrome of the leg. Retrospectively, at least three of this potential risk factors were present in the described case.

As demonstrated by Halliwill et al. [8], lithotomy position decreases the lower extremity perfusion; particularly the systolic blood pressure to lower limbs may be significantly reduced and insufficient to provide satisfactory oxygenation to tissues. Most importantly, cadaver studies demonstrated that the lithotomy position can severely kink femoral vessels (and nerves) due to the flexion and abduction/external rotation of the thigh.

Depending on the duration of surgery and the different degree of hip angulation and legs height positioning, the alterations of the blood pressure and the distortion of the neurovascular bundles may reduce the blood flow and the perfusion to the lower limb, causing blood stasis possibly contributing to the formation of the arterial thrombus. Moreover, pressure monitoring at the radial artery in trendelenburg/lithotomy may be artificially high; we can suppose reasonably that severe hypoperfusion of the lower extremities occurred in our patient, despite an apparently ideal pressure monitoring. Then, being the sub-geniculate area sterile-draped and covered, a potential initial clinical sign of ischemia (e.g., thermal gradient of the skin), was more difficult to notice during the operation. Instead, the perioperative monitoring of pedal blood pressure by intraarterial cannulation or pulse oximeter on lower limbs may have been convenient and should be performed for high-risk patients. This is a relatively simple procedure and provides an immediate reaction when there is decreased flow to the foot [4].

Retrospectively analyzing our case experience, we found further factors that may have contributed to acute arterial ischemia 
and are worth being noticed: recent poly-trauma, previous DVT, thrombocythemia and inflammatory state at blood test [11].

Plastic surgeons should always consider the potential risks of prolonged lithotomy position. Despite less common than DVT and compartmental syndrome, arterial thrombosis is a potentially devastating complication and plastic surgeons should be aware of the danger, as the risk of focusing on the technical aspects of the reconstructive procedures while losing sight of the bigger picture. Careful preoperative evaluation of patient is of paramount importance in order to identify patient at risk and implement all preventive measures. Postoperatively, particular care should be used so as to recognize promptly any early symptoms of limb ischemia, in order to carry out the required clinical treatments.

\section{NOTES}

\section{Conflict of interest}

No potential conflict of interest relevant to this article was reported.

\section{Ethical approval}

The study was performed in accordance with the principles of the Declaration of Helsinki. Written informed consent was obtained.

\section{Patient consent}

The patient provided written informed consent for the publication and the use of his images.

\section{Author contribution}

Conceptualization: G Sapino, PG di Summa. Visualization: S Deglise, W Raffoul, PG di Summa. Writing - original draft: G Sapino. Writing - review \& editing: G Sapino, PG di Summa.

\section{ORCID}

Gianluca Sapino

Sebastien Deglise

Wassim Raffoul https://orcid.org/0000-0003-2716-8607

https://orcid.org/0000-0001-8244-952X

https://orcid.org/0000-0002-3524-0077
Pietro G. di Summa https://orcid.org/0000-0002-1431-4479

\section{REFERENCES}

1. Bauer EC, Koch N, Janni W, et al. Compartment syndrome after gynecologic operations: evidence from case reports and reviews. Eur J Obstet Gynecol Reprod Biol 2014;173:712.

2. Gelder C, McCallum AL, Macfarlane AJR, et al. A systematic review of mechanical thromboprophylaxis in the lithotomy position. Surgeon 2018;16:365-71.

3. Yanazume S, Yanazume Y, Iwamoto I, et al. Severe leg compartment syndrome associated with dorsal lithotomy position during radical hysterectomy. J Obstet Gynaecol Res 2006;32:610-2.

4. Sahara K, Ishibe A, Yabuno T, et al. Acute iliac arterial thrombosis during laparoscopic abdominoperineal resection.J Surg Case Rep 2019;2019:rjz020.

5. Canterbury TD, Wheeler WE, Scott-Conner CE. Effects of the lithotomy position on arterial blood flow in the lower extremities. WV Med J 1992;88:100-1.

6. di Summa PG, Tremp M, Meyer Zu Schwabedissen M, et al. The combined pedicled anterolateral thigh and vastus lateralis flap as filler for complex perineal defects. Ann Plast Surg 2015;75:66-73.

7. Angermeier KW, Jordan GH. Complications of the exaggerated lithotomy position: a review of 177 cases. J Urol 1994;151:866-8.

8. Halliwill JR, Hewitt SA, Joyner MJ, et al. Effect of various lithotomy positions on lower-extremity blood pressure. Anesthesiology 1998;89:1373-6.

9. Nakamura K, Aoki H, Hirakawa T, et al. Compartment syndrome with thrombosis of common iliac artery after gynecologic surgery. Obstet Gynecol 2008;112(2 Pt 2):486-8.

10. Kikuchi T, Maeda H. Two cases of compartment syndrome of the lower extremities during surgery for gynecological malignancies. J Anesth 2016;30:481-5.

11. Creager MA, Kaufman JA, Conte MS. Clinical practice: acute limb ischemia. N Engl J Med 2012;366:2198-206. 